\title{
Out-of-band emission suppression techniques based on a generalized OFDM framework
}

\author{
Zihao You*, Juan Fang and I-Tai Lu
}

\begin{abstract}
Orthogonal frequency division multiplexing (OFDM)-based cognitive radio (CR) systems suffer from the large out-of-band emission (OOBE) that may interfere with other users. Since most existing OFDM OOBE suppression schemes are derived on the base of an original OFDM system without any other scheme, we first propose a generalized OFDM framework that is capable of describing these schemes no matter whether any one or more of the schemes is applied. Then, according to the place where these schemes are implemented in our framework, they are classified into three groups, namely symbol mapping techniques, precoding techniques, and time-domain techniques. Finally, based on the proposed framework, we propose three new schemes by combining a precoding scheme named singular value decomposition (SVD) precoding with three other schemes from the three groups, namely spectral precoding, $\mathrm{N}$-continuous symbol mapping, and filtering. Numerical results show the power spectral density (PSD), peak-to-average power ratio (PAPR), and bit error rate (BER) performances of the three proposed schemes. Since the individual schemes have complementary characteristics, the three proposed combined schemes are constructed to maintain the merits and avoid the drawbacks of the individual schemes involved. Thus, it is demonstrated that the proposed framework can be employed to develop other new combined OOBE suppression schemes tailoring to some specific practical needs.
\end{abstract}

Keywords: OFDM; Cognitive radio; Out-of-band emission

\section{Introduction}

Cognitive radio (CR), as a promising solution to the spectrum congestion problem brought by the rapid increasing number of wireless communication techniques and devices, has drawn significant attention recently (for example, in [1-3]). CR realizes dynamic access to the spectrum resources which could be non-contiguous and be available at certain times and locations. The availability depends on whether these spectrum resources are occupied by the licensed users (LUs). The secondary users (SUs) are required to be able to operate in the vacant parts of the allocated spectrum which are not occupied by the LUs. Therefore, a waveform that can flexibly control the spectral shape of the transmitted signal is needed so that SUs and LUs share the same allocated spectrum.

Orthogonal frequency division multiplexing (OFDM) technology, which divides the total bandwidth into several orthogonal sub-bands, provides the flexibility of

\footnotetext{
* Correspondence: zyou01@students.poly.edu

Department of ECE, NYU WIRELESS, Polytechnic Institute of NYU, 6 Metrotech Center, Brooklyn, NY 11201, USA
}

deactivating specific subcarriers simply by using zeros as the corresponding input values. Because of this, OFDM has been considered as a candidate for CR in the first cognitive radio-based standard IEEE 802.22 [4]. OFDM has several favorable properties like high spectrum efficiency, robustness to channel fading, multipath delay spread tolerance, efficient fast Fourier transform (FFT) implementation, etc. (for example, in [5-7]). However, one of the major problems is the large sidelobes of all OFDM subcarriers. As a result, OFDM-based CR systems suffer from large out-of-band emission (OOBE) that may interfere with other bands occupied by LUs.

Various schemes have been proposed to reduce the OOBE of OFDM-based CR systems. They can be classified into two categories: the time-domain schemes and the frequency-domain schemes. The time-domain schemes include filtering [8], windowing [9-11], etc., where the OOBE reduction processes are carried out after the inverse fast Fourier transform (IFFT). The frequency-domain schemes include carrier cancellation (CC) [12], constellation extension 
(CE) [13], precoding [14-18], etc., where the OOBE reduction processes are carried out before the IFFT.

Since the existing OOBE suppression techniques suffer from various penalties, proper combinations of two or more schemes might enhance the overall OOBE suppression and mitigate their penalties. However, since most OOBE suppression schemes are derived on the base of an original OFDM system without the implementation of any scheme, it may be challenging to find a systematic way to implement more than one scheme in one system simultaneously. It may also be hard to tell whether these different schemes are contradictive, compatible, or complementary. Therefore, in this paper, we introduce a generalized OFDM framework. For all the OOBE suppression schemes that we have investigated, no matter whether any one or more of them is implemented on an OFDM system, that system can be always represented by our proposed framework. As all the designs are based on the same unified system now, it is convenient to develop new OOBE suppression schemes by combining different existing schemes. The combinations of different techniques may not be trivial since some of these techniques will change each other's features when they are implemented together. Thus, if the techniques being combined do affect each other, special designs need to be made such that each constituent technique of the combined scheme can retain its desirable functionalities. On the other hand, if the techniques being combined do not affect each other, the constituent techniques of the combined scheme can then be implemented one after another in a decentralized way. (For convenience, this latter process will be called 'combining but no merging' in this paper.)

Using the proposed unified framework, we also classify some of the existing OFDM OOBE suppression techniques into three groups depending on the positions where they are implemented in our framework. Here, the time-domain schemes remain in one group, but the frequency-domain schemes are further divided into two groups: the symbol mapping techniques and the precoding techniques. To demonstrate the convenience of using the proposed framework, we propose three new types of hybrid OOBE suppression schemes by selecting a scheme from each group and combining it with the precoding scheme in $[14,15]$ (will be called singular value decomposition (SVD) precoding due to the principle of its design). The three selected schemes are spectral precoding [16], $N$-continuous precoding [17] (will be called $N$-continuous symbol mapping in this paper due to our classification), and filtering [8]. It is obvious that spectral precoding, $N$ continuous symbol mapping, and filtering belong to the precoding, symbol mapping, and time-domain techniques, respectively. Since SVD precoding and each of the three selected schemes have complementary characteristics (which will be explained later in the paper), the three proposed combined schemes maintain the merits and avoid (or reduce) the drawbacks of the individual schemes involved. It will be shown that special designs need to be made for combining SVD precoding with spectral precoding or $\mathrm{N}$-continuous symbol mapping. As for combining SVD precoding and filtering, a simple combining but no merging process is sufficient. Numerical results show the power spectral density (PSD) performance of the three proposed schemes for two scenarios, one with cyclic prefix (CP) and one without CP. It is shown that the proposed combined schemes indeed outperform their individual constituent schemes used, which also implies that the same goal can be achieved by the combined schemes with smaller cost than the individual schemes. Other performances like peak-to-average power ratio (PAPR) and bit error rate (BER) are briefly discussed. Practical considerations about complexity, numerical precision limit due to hardware constraints, and OOBE regrowth due to nonlinear elements are also addressed.

The outline of this paper is given below. Section 2 presents the generalized OFDM framework. Section 3 briefly investigates some existing OOBE suppression techniques and represents them using the proposed framework. Section 4 gives three examples of combining different existing schemes. Numerical results are shown in Section 5. Conclusion is made in Section 6. Notations are as follows. All boldface letters indicate vectors (lowercase) or matrices (uppercase). Matrix I means an identity matrix of proper dimension. Matrix (vector) 0 means a zero matrix (vector) of proper dimension. $\mathbf{A}^{H}$ stands for the conjugate transpose of $\mathbf{A}$.

\section{Generalized OFDM framework}

The block diagram of a generalized OFDM framework is shown in Figure 1. As shown in the figure, the source bit stream is mapped into a symbol stream by PSK/ QAM modulation and goes through the serial-to-parallel $(\mathrm{S} / \mathrm{P})$ conversion. Let $\mathbf{d}_{l}=\left[\begin{array}{llll}d_{1, l} & d_{2, l} & \ldots & d_{K, l}\end{array}\right]^{T}$ denote the data vector in the $l$ th time slot. $K$ is the length of each vector. Then, each vector first goes through the 'freqdomain approach' block, where the frequency-domain OOBE suppression schemes (including precoding and symbol mapping techniques) could be applied. Let the output of the freq-domain approach block be

$$
\mathbf{b}_{l}=\mathbf{G c}_{l},
$$

where $\mathbf{G}$ can be viewed as a precoding matrix and $\mathbf{c}_{l}$ is an output of a symbol mapping from $\mathbf{d}_{l}$ (Note that the dimension of $\mathbf{b}_{l}$ can be greater or equal to that of $\mathbf{c}_{l}$ and the dimension of $\mathbf{c}_{l}$ can be greater or equal to that of $\mathbf{d}_{l}$.) The symbol mapping could simply let the output be the same as the input, or expand the constellations, choose from multiple available vectors, add a perturbation 


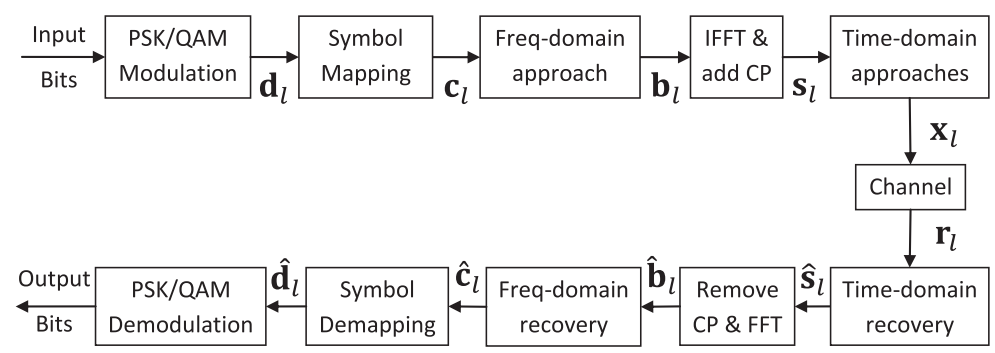

Figure 1 Block diagram of a generalized precoded-OFDM system.

vector, etc. Then, the frequency-domain output $\mathbf{b}_{l}$ goes through the IFFT and possibly the CP insertion process. The process can be represented by left-multiplying $\mathbf{b}_{l}$ by another matrix F, i.e.,

$$
\mathbf{s}_{l}=\mathbf{F G} \mathbf{c}_{l}
$$

Finally, $\mathbf{s}_{l}$ goes through the 'time-domain approach' block, where the time-domain OOBE suppression schemes could be applied. Let the output of the 'time-domain approach' block be

$$
\mathbf{x}_{l}=\mathbf{H F G} \mathbf{c}_{l}+\boldsymbol{\varepsilon}_{l},
$$

where $\mathbf{H}$ can be used to realize the windowing, filtering, etc., and $\boldsymbol{\varepsilon}_{l}$ is a time-domain perturbation vector, which could be used to represent some non-linear schemes.

At the receiver, the 'time-domain recovery' process is applied to the received signal $\mathbf{r}_{l}$ first. Let the output be

$$
\hat{\mathbf{s}}_{l}=\widehat{\mathbf{H}}\left(\mathbf{r}_{l}-\hat{\boldsymbol{\varepsilon}}_{l}\right)
$$

where $\hat{\boldsymbol{\varepsilon}}_{l}$ is the estimation of $\boldsymbol{\varepsilon}_{l}$ and $\widehat{\mathbf{H}} \mathbf{H}=\mathbf{I}$. Then, we remove the $\mathrm{CP}$ (if inserted at the transmitter) from $\hat{\mathbf{s}}_{l}$ and make it go through the FFT block. The output is

$$
\hat{\mathbf{b}}_{l}=\widehat{\mathbf{F}} \widehat{\mathbf{H}}\left(\mathbf{r}_{l}-\hat{\boldsymbol{\varepsilon}}_{l}\right),
$$

where $\widehat{\mathbf{F}} \mathbf{F}=\mathbf{I}$. Afterwards, the 'freq-domain recovery' process is applied. Let

$$
\hat{\mathbf{c}}_{l}=\widehat{\mathbf{G}} \widehat{\mathbf{F}} \widehat{\mathbf{H}}\left(\mathbf{r}_{l}-\hat{\boldsymbol{\varepsilon}}_{l}\right)
$$

where $\widehat{\mathbf{G}} \mathbf{G}=\mathbf{I}$. Finally, the decision vector $\hat{\mathbf{d}}_{l}$ is derived by an inverse symbol mapping from $\hat{\mathbf{c}}_{l}$.

\section{Some existing OOBE suppression techniques}

In this section, we briefly review some existing OOBE suppression techniques. According to how the techniques are implemented in the proposed framework in Section 2, they are categorized into three groups, which are the techniques represented by the precoding matrices, the techniques represented by the symbol mapping, and the techniques represented by the time-domain approaches.

\subsection{Techniques represented by the precoding matrices}

For this group of techniques, since only the precoding matrix is used, then at the transmitter, we have $\mathbf{c}_{l}=\mathbf{d}_{l}, \boldsymbol{\varepsilon}_{l}=$ $\mathbf{0}$, and $\mathbf{H}=\mathbf{I}$. The parameters at the receiver can be set accordingly. The simplest way in this group to suppress the OOBE is to deactivate some subcarriers on the edges as the guard subcarriers (GS) [9]. Suppose the number of subcarriers for transmitting data is $K$ and the number of guard subcarriers on each edge is $(N-K) / 2$. Then, the precoding matrix representing the GS method is

$$
\mathbf{G}=\left[\begin{array}{c}
\mathbf{0}_{\frac{N-K}{2} \times K} \\
\mathbf{I}_{K \times K} \\
\mathbf{0}_{\frac{N-K}{2} \times K}^{2}
\end{array}\right] .
$$

Another method, called CC [12], also deactivates the subcarriers on the edge. But it designs the input of these subcarriers within a defined power threshold to minimize the radiation at certain frequencies, which are usually assigned to LUs. So, the CC matrix is similar to (7), but the two $\mathbf{0}$ matrices in (7) are replaced with other entries. Subcarrier weighting (SW) method [19] multiplies all the data subcarriers with some real weighting coefficients. Therefore, the SW matrix is a diagonal matrix, and the weighting coefficients are the diagonal entries. Another precoding method that has better OOBE suppression effect than SW is proposed in $[14,15]$, where the matrix is designed to ensure that any precoded data is in the null space of the matrix representing the data's frequency-domain components at some selected out-of-band frequencies. This scheme is called SVD precoding in this paper due to the principle of its design. The coding rate of SVD precoding is less than 1 , which means that the spectrum efficiency is decreased. Another precoding scheme called spectral precoding is proposed in [16]. It uses new orthogonal basis sets to replace the rectangular pulse for each conventional OFDM symbol so that the new sidelobes fall off faster. The spectrum efficiency is also reduced due to the limited number of available basis sets when the inband range is fixed. 
The group A techniques mentioned above are summarized in Table 1.

\subsection{Techniques represented by the symbol mapping}

For this group of techniques, since only the symbol mapping is used, then at the transmitter, we have $\boldsymbol{\varepsilon}_{l}=\mathbf{0}$, $\mathbf{G}=\mathbf{I}$, and $\mathbf{H}=\mathbf{I}$. (Note that $\mathbf{G}$ and $\mathbf{H}$ may have different dimensions.) The parameters at the receiver can be set accordingly. A straightforward method called multiplechoice sequence (MCS) is proposed in [20]. The data symbol is element-wise produced by a set of vectors. The product that yields the lowest OOBE is chosen as the mapping output. The information that shows which vector is chosen should also be known by the receiver as the overhead for each symbol. Another method is called CE [13]. It increases the constellation points so that there are multiple choices for each subcarrier input. The best combination of all possible inputs is chosen to minimize the OOBE. The minimum distance of the constellation points is reduced as the points increase, so the BER performance would become worse. Another scheme called $N$-continuous symbol mapping is proposed in [17]. Instead of designing a precoding matrix, $\mathrm{N}$-continuous symbol mapping designs a perturbation vector depending on the current and the previous symbols so that the corresponding time-domain symbols have continuous values and derivatives everywhere. Since the perturbation vector may not be correctly estimated at the receiver, an iterative decoder is used.

The group B techniques mentioned above are summarized in Table 2.

\subsection{Techniques represented by the time-domain approaches}

For this group of techniques, since only the time-domain approaches are used, then at the transmitter, we have $\mathbf{c}_{l}=\mathbf{d}_{l}$,

Table 1 OOBE suppression techniques group A, principles and drawbacks included

\begin{tabular}{|c|c|}
\hline $\begin{array}{l}\text { Group A } \\
\left(c_{l}=d_{l}, \varepsilon_{l}=0, H=I\right)\end{array}$ & Principle and drawbacks \\
\hline \multirow[t]{2}{*}{ GS } & $\begin{array}{l}\text { Principle: reserved subcarrier on the edges with } \\
\text { zero input }\end{array}$ \\
\hline & Drawback: spectrum efficiency loss \\
\hline \multirow[t]{2}{*}{$\mathrm{CC}$} & $\begin{array}{l}\text { Principle: reserved subcarrier on the edges with } \\
\text { non-zero input }\end{array}$ \\
\hline & $\begin{array}{l}\text { Drawback: spectrum efficiency loss; data-dependent } \\
\text { design }\end{array}$ \\
\hline \multirow[t]{2}{*}{ SW } & $\begin{array}{l}\text { Principle: different subcarriers assigned with } \\
\text { different coefficients }\end{array}$ \\
\hline & Drawback: data-dependent design \\
\hline \multirow[t]{2}{*}{ Precoding } & $\begin{array}{l}\text { Principle: a precoding matrix (different purposes } \\
\text { for different approaches) }\end{array}$ \\
\hline & Drawback: spectrum efficiency loss \\
\hline
\end{tabular}

Table 2 OOBE suppression techniques group B, principles and drawbacks included

\begin{tabular}{ll}
$\begin{array}{l}\text { Group } \mathbf{B} \\
\left(\boldsymbol{\varepsilon}_{\boldsymbol{l}}=\mathbf{0}, \mathbf{G}=\mathbf{I}, \mathbf{H}=\mathbf{I}\right)\end{array}$ & Principle and drawbacks \\
\hline MCS & Principle: multiple streams for selection \\
& Drawback: overhead (spectrum efficiency loss) \\
$\mathrm{CE}$ & Principle: constellation expansion \\
& $\begin{array}{l}\text { Drawback: enlarged transmit power (BER } \\
\text { performance degradation) }\end{array}$ \\
$\mathrm{N}$-continuous & Principle: continuous signal and derivatives of signal \\
& Drawback: iterative decoder \\
\hline
\end{tabular}

and $\mathbf{G}=\mathbf{I}$. The windowing method including several kinds of window functions is proposed in [9-11]. Since the window function is directly multiplied to the signal after IFFT, $\varepsilon_{l}=\mathbf{0}, \mathbf{H}$ is a diagonal matrix and the values of its diagonal entries depend on the selection of the window function. The matrix $\mathbf{F}$ might be modified in some cases that both cyclic suffix (CS) and CP are required so that the signal is as long as the window. Another method, called adaptive symbol transition (AST), also extends the signal by inserting extra samples within a defined power threshold between two transmission blocks to minimize the radiation of these two blocks at certain frequencies [21]. Therefore, $\mathbf{H}$ for ASC method has the similar structure as the precoding matrix for CC method in [12]. However, the solution of the extended signal is non-linear, so the vector $\boldsymbol{\varepsilon}_{l}$ is required along with $\mathbf{H}$ to represent the transmitted signal. The filtering method is proposed in [8]. Since the transmit signal is the convolution of the filter and the data in time-domain, thus, $\mathbf{H}$ for the filtering method is a convolution matrix which can be derived from the filter impulse response and $\boldsymbol{\varepsilon}_{l}=\mathbf{0}$.

The group $C$ techniques mentioned above are summarized in Table 3.

\section{Combinations of existing OOBE suppression methods}

From the classification, we see that when a single original scheme is expressed using the framework, many matrices in (1) to (6) are zero or identity matrices. With the help of the generic structure, the process of combining some of

Table 3 OOBE suppression techniques group C, principles and drawbacks included

\begin{tabular}{|c|c|}
\hline $\begin{array}{l}\text { Group C } \\
\left(c_{I}=d_{l}, G=I\right)\end{array}$ & Principle and drawbacks \\
\hline \multirow[t]{2}{*}{$\overline{\text { AST }}$} & Principle: inserting samples between two time blocks \\
\hline & Drawback: spectrum efficiency loss; data-dependent design \\
\hline \multirow[t]{2}{*}{ Windowing } & Principle: some window function applied in time domain \\
\hline & Drawback: extension of symbol (spectrum efficiency loss) \\
\hline \multirow[t]{2}{*}{ Filtering } & Principle: some filter applied in time domain \\
\hline & Drawback: extension of symbol (spectrum efficiency loss) \\
\hline
\end{tabular}


the different methods can be viewed as the process of replacing some of the zero or identity matrices with others. It would be straightforward to see how the combination works after the replacement. For the methods which belong to the same group, however, the framework does not help design the combination except for the classification. In this section, we propose three hybrid schemes which combine the SVD precoding scheme in [15], which belongs to group A in Section 3, with the other three schemes which belong to the three groups, respectively.

Define the $l$ th continuous time-domain transmit signal $x_{l}(t)$ for a given $\mathbf{b}_{l}$ in a CP-OFDM system as

$$
x_{l}(t)=\sum_{i=1}^{N} b_{i, l} e^{j 2 \pi \frac{f_{i}}{T_{d}} t}, \quad-T_{\mathrm{CP}} \leq t<T_{d} .
$$

Here $N$ is the number of subcarriers, $b_{i, l}$ is the $i$ th element of $\mathbf{b}_{l}, T_{d}$ is the OFDM symbol length (without $\mathrm{CP}), T_{\mathrm{CP}}$ is the CP length, and $f_{i}(i=1,2, \ldots, N)$ is the $i$ th subcarrier's frequency index. Then, $T=T_{\mathrm{CP}}+T_{d}$ is the entire block length for each time-domain transmission block.

Note that the frequency component of $x_{l}(t)$ is

$$
X_{l}(f)=\sum_{i=1}^{N} b_{i, l} P_{f_{i}}(f),
$$

where the frequency response of the $i$ th subcarrier

$$
P_{f_{i}}(f)=\frac{\mathrm{e}^{j \pi\left(\frac{f_{i}}{T_{d}}-f\right)\left(T_{d}-T_{\mathrm{CP}}\right)}}{\pi\left(\frac{f_{i}}{T_{d}}-f\right) T} \sin \left(\pi\left(\frac{f_{i}}{T_{d}}-f\right) T\right) .
$$

Suppose that we want to minimize the radiation power at the out-of-band frequencies $h_{1}, h_{2}, \ldots, h_{M}$ by designing an SVD precoding matrix $\mathbf{G}^{S}$ so that $\mathbf{b}_{l}=\mathbf{G}^{S} \mathbf{c}_{l}$ knowing that $\mathbf{c}_{l}=\mathbf{d}_{l}$. Denote the frequency sampling vector $\mathbf{X}_{l}=\left[\begin{array}{llll}X_{l}\left(h_{1}\right) & X_{l}\left(h_{2}\right) & \ldots & X_{l}\left(h_{M}\right)\end{array}\right]^{T}$, then

$$
\mathbf{X}_{l}=\mathbf{P G}^{S} \mathbf{d}_{l}, \mathbf{P}=\left[\begin{array}{ccc}
P_{f_{1}}\left(h_{1}\right) & \cdots & P_{f_{N}}\left(h_{1}\right) \\
\vdots & \ddots & \vdots \\
P_{f_{1}}\left(h_{M}\right) & \cdots & P_{f_{N}}\left(h_{M}\right)
\end{array}\right] .
$$

To minimize $\left\|\mathbf{X}_{l}\right\|$ regardless of $\mathbf{d}_{l}$, perform the SVD of $\mathbf{P}$ as

$$
\mathbf{P}=\mathbf{U} \boldsymbol{\Sigma} \mathbf{V}^{H},
$$

where $\mathbf{U}$ is an $M \times M$ unitary matrix, $\boldsymbol{\Sigma}$ is a diagonal $M \times N$ matrix containing the singular values of $\mathbf{P}$ in non-increasing order, and $\mathbf{V}$ is an $N \times N$ unitary matrix whose columns are $\mathbf{v}_{1}, \mathbf{v}_{2}, \cdots, \mathbf{v}_{N}$. The $N \times K$ SVD precoding matrix is then chosen as

$$
\mathbf{G}^{S}=\left[\begin{array}{llll}
\mathbf{v}_{N-K+1} & \mathbf{v}_{N-K+2} & \ldots & \mathbf{v}_{N}
\end{array}\right] .
$$

Define $R=N-K$ as the coding redundancy, and $K / N$ is the coding rate. If $R \geq M$, then $\left\|\mathbf{X}_{l}\right\|=0$ for any arbitrary $\mathbf{d}_{l}$ because $\mathbf{b}_{l}$ is always in the null space of $\mathbf{P}$. Since $\mathbf{G}^{S}$ is a semi-unitary matrix, $\left(\mathbf{G}^{S}\right)^{H} \mathbf{G}^{S}=\mathbf{I}$ and the decoding matrix is simply $\left(\mathbf{G}^{S}\right)^{H}$.

Since the design of $\mathbf{G}^{S}$ does not depend on data $\mathbf{d}_{l}$, SVD precoding is a data-independent scheme.

\subsection{Combination of SVD precoding [15] and spectral precoding [16] (two group A methods)}

It is pointed out in [16] that the rectangularly pulsed OFDM possesses discontinuous pulse edges and exhibits relatively large power spectral sidelobes which fall off as $f^{2}$, while the continuous phase OFDM signals exhibit relatively small power spectral sidelobes which fall off as $f^{4}$ and can thus provide much higher spectrum efficiency than the traditional rectangularly pulsed OFDM signal. Two families of new basis sets which satisfy the continuous phase requirement are introduced, named as family $W$ and family $V$.

The corresponding precoded OFDM structure that is used to construct OFDM signals using the basis sets along with the arbitrary input data is given below. The entries of the family $W_{L}$-based precoding matrix $\mathbf{G}^{W_{L}}$ are defined as

$$
g_{N\left(1-2^{1-u}\right)+n, n 2^{u}+v}^{W_{L}}=2^{-\frac{u}{2}}(-1)^{1+\psi_{u, v}}, n \in\left[0, \frac{N}{2^{u}}-1\right] \text { and } v \in\left[0,2^{u}-1\right]
$$

for $u=1,2, \ldots, L$. In (14), $\psi_{u, v}$ is the sum of the most and least significant bits in the binary representation (in bits) of the modulo- $2^{u}$ value of $v$ when $u \geq 2$ and $\psi_{1, v}=1$ by default. All other entries are equal to 0 . The entries of the family $V_{L}$-based precoding matrix $\mathbf{G}^{V_{L}}$ are defined as

$$
g_{N\left(1-2^{1-u}\right)+n, n+\frac{N}{2^{u} v}}^{V_{L}}=2^{-\frac{u}{2}} \phi_{u, v}, n \in\left[0, \frac{N}{2^{u}}-1\right] \text { and } v \in\left[0,2^{u}-1\right],
$$

for $u=1,2, \ldots, L$. In (15), $\phi_{u, v}=1$ if $u=\log _{2} N$ and $\phi_{u, v}=(-1)^{\zeta_{v}}$ otherwise, where $\zeta_{v}$ represents the least significant bit in the binary representation of $v$. All other entries are equal to $0 . L$ in (14) and (15) is a parameter that determines the coding rate, which is equal to $1-2^{-L}, L \in$ $\left[1, \log _{2} N\right]$. Since $\mathbf{G}^{W_{L}}$ and $\mathbf{G}^{V_{L}}$ are both semi-unitary matrices, then the decoding matrices are their conjugate transposes.

Since both SVD precoding and spectral precoding use the precoding matrices (using $\mathbf{G}^{S}$ and $\mathbf{G}^{W_{L}}$ as example), we can combine them by defining either $\mathbf{G}=\mathbf{G}^{S} \mathbf{G}^{W_{L}}$ or $\mathbf{G}=\mathbf{G}^{W_{L}} \mathbf{G}^{S}$. The former way is not good because the continuous phase property brought by $\mathbf{G}^{W_{L}}$ cannot be maintained after it is left-multiplied by $\mathbf{G}^{S}$, which means $\mathbf{G}^{W_{L}}$ would be useless. On the other hand, by choosing the latter way, we can keep the continuous phase property. Furthermore, we can modify the derivation of $\mathbf{G}^{S}$ in 
(11) to (13) to keep the null space property in the new hybrid method. Here are the steps of the combined design. First, using (14), design the spectral precoder as

$$
\overline{\mathbf{G}}^{W_{L}}=\mathbf{G}^{W_{L}}
$$

without considering the SVD precoder $\mathbf{G}^{S}$. Second, perform the SVD of $\mathbf{Q}=\mathbf{P G}^{W_{L}}$, i.e., let

$$
\mathbf{Q}=\mathbf{P G}^{W_{L}}=\overline{\mathbf{U}} \bar{\Sigma} \overline{\mathbf{V}}^{H}
$$

Next, using (13) and (17), we have the modified SVD precoder

$$
\overline{\mathbf{G}}^{S}=\left[\begin{array}{llll}
\overline{\mathbf{v}}_{N-K+1} & \overline{\mathbf{v}}_{N-K+2} & \ldots & \overline{\mathbf{v}}_{N}
\end{array}\right] .
$$

Finally, let the combined SVD and spectral precoder

$$
\mathbf{G}=\overline{\mathbf{G}}^{W_{L}} \overline{\mathbf{G}}^{S}, \widehat{\mathbf{G}}=\left(\overline{\mathbf{G}}^{W_{L}} \overline{\mathbf{G}}^{S}\right)^{H}
$$

Note that the transmitted signal $\mathbf{x}_{l}$ for spectral precoding in [16] is the real part of the IFFT output, while the complex output is used for SVD precoding in [15]. In this paper, the complex output is used. The matched dimensions of $\mathbf{G}^{S}$ and $\mathbf{G}^{W_{L}}$ for a given dimension of $\mathbf{G}$ can be found by trying all the possible dimension pairs so that the best OOBE suppression can be achieved.

On the other hand, what will happen if SVD precoding and spectral precoding are implemented one after another in a decentralized way that each precoding matrix is designed without knowing the existence of the other matrix? Since both two schemes are group A schemes, the transmitted signal $\mathbf{x}_{l}$ can be either $\mathbf{F G}^{S} \mathbf{G}^{W_{L}} \mathbf{d}_{l}$ or $\mathbf{F G}^{W_{L}} \mathbf{G}^{S} \mathbf{d}_{l}$. For the former case, $\mathbf{G}^{S} \mathbf{G}^{W_{L}}$ is not able to construct the desired basis set. For the latter case, $\mathbf{P G}^{S} \mathbf{G}^{W_{L}}$ is no longer $\mathbf{0}$. In other words, the effect of combining but no merging will be the same as implementing just one of them, thus making the spectrum efficiency loss from the other scheme completely useless.

4.2 Combination of SVD precoding [15] and $\mathrm{N}$-continuous symbol mapping [17] (group A and group B)

It is said earlier in [16] that one of the causes of high OOBE is the discontinuity of time-domain signals. Since the signal is always continuous everywhere within a CPOFDM symbol, we just need to make the value and its first to Jth derivatives on the left edge of every CPOFDM symbol equal to those on the right edge of its previous symbol. The problem is expressed as adjusting the symbol mapping from $\mathbf{d}_{l}$ to $\mathbf{c}_{l}$ so that

$$
\left.\frac{d^{j}}{d t^{j}} x_{l}(t)\right|_{t=-T_{\mathrm{CP}}}=\left.\frac{d^{j}}{d t^{j}} x_{l-1}(t)\right|_{t=T_{d}}(j=0,1, \ldots, J) .
$$

In [17], only a perturbation vector $\boldsymbol{\delta}_{l}^{N}$ is used to satisfy (20). Let $\mathbf{c}_{l}=\mathbf{d}_{l}+\boldsymbol{\delta}_{l}^{N}$. Then, the problem is equivalent to finding a $\boldsymbol{\delta}_{l}^{N}$ so that

$$
\mathbf{A} \Phi \mathbf{c}_{l}=\mathbf{A c}_{l-1},
$$

where $\boldsymbol{\Phi}=\operatorname{Diag}\left(e^{j 2 \pi \frac{-T_{\mathrm{CP}}}{T_{d}} f_{1}}, e^{j 2 \pi \frac{-T_{\mathrm{CP}}}{T_{d}} f_{2}}, \ldots, e^{j 2 \pi \frac{-T_{\mathrm{CP}}}{T_{d}} f_{N}}\right)$ and $\mathbf{A}=\left[\begin{array}{cccc}1 & 1 & \cdots & 1 \\ f_{1} & f_{2} & \cdots & f_{N} \\ \vdots & \vdots & \ddots & \vdots \\ f_{1}^{J} & f_{2}^{J} & \cdots & f_{N}^{J}\end{array}\right]$

Since $\mathbf{A}$ is a $(J+1) \times N$ matrix and $N$ is usually much larger than $(J+1)$, the solution of $\boldsymbol{\delta}_{l}^{N}$ is not unique. Among these solutions, the solution of the least magnitude is found by introducing the Moore-Penrose pseudoinverse of $\mathbf{A} \Phi$, denoted as $(\mathbf{A} \Phi)^{+}$. Then, that solution is

$$
\boldsymbol{\delta}_{l}^{N}=\left\{\begin{array}{c}
(\mathbf{A} \boldsymbol{\Phi})^{+} \mathbf{A c}_{l-1}-(\mathbf{A} \boldsymbol{\Phi})^{+} \mathbf{A} \boldsymbol{\Phi} \mathbf{d}_{l}, l>1 \\
\mathbf{0}, l=1
\end{array}\right.
$$

At the receiver, it is supposed that there is no channel effect or noise, $\hat{\mathbf{c}}_{l}=\mathbf{c}_{l}$ can be correctly recovered after FFT process. $\boldsymbol{\delta}_{l}^{N}$ needs to be correctly estimated so that it can be removed from $\hat{\mathbf{c}}_{l}$ to recover $\mathbf{d}_{l}$. However, from (22) we know that $\boldsymbol{\delta}_{l}^{N}$ depends on $\mathbf{d}_{l}$. Therefore, an iterative decoder is needed.

In the $i$ th $(i \geq 1)$ iteration, the estimated perturbation vector $\hat{\boldsymbol{\delta}}_{l}^{N,(i)}$ is

$$
\hat{\boldsymbol{\delta}}_{l}^{N,(i)}=(\mathbf{A} \boldsymbol{\Phi})^{+} \mathbf{A} \boldsymbol{\Phi} \hat{\mathbf{c}}_{l}-(\mathbf{A} \boldsymbol{\Phi})^{+} \mathbf{A} \boldsymbol{\Phi} \overline{\mathbf{d}}_{l}^{(i-1)},
$$

$\overline{\mathbf{d}}_{l}^{(i-1)}$ in (23) is supposed to be known. Next, the $i$ th iteration decision vector $\hat{\mathbf{d}}_{l}^{(i)}$ is derived as

$$
\begin{aligned}
\hat{\mathbf{d}}_{l}^{(i)} & =\hat{\mathbf{c}}_{l}-\hat{\boldsymbol{\delta}}_{l}^{N,(i)} \\
& =\left(\mathbf{I}-(\mathbf{A \Phi})^{+} \mathbf{A \Phi}\right) \hat{\mathbf{c}}_{l}+(\mathbf{A \Phi})^{+} \mathbf{A} \boldsymbol{\Phi} \overline{\mathbf{d}}_{l}^{(i-1)} .
\end{aligned}
$$

If it is not the last iteration, then $\overline{\mathbf{d}}_{l}^{(i)}(i \geq 1)$ is chosen from all possible frequency-domain symbol vectors (In other words, every entry of $\overline{\mathbf{d}}_{l}^{(i)}$ is a constellation point.) to minimize $\left\|\hat{\mathbf{d}}_{l}^{(i)}-\overline{\mathbf{d}}_{l}^{(i)}\right\|$. The initial vector $\overline{\mathbf{d}}_{l}^{(0)}=\mathbf{0}$.

Although $\boldsymbol{\delta}_{l}^{N}$ depends on $\mathbf{c}_{l-1}$ and $\mathbf{d}_{l}$, the matrices $(\mathbf{A} \boldsymbol{\Phi})^{+} \mathbf{A}$ and $(\mathbf{A} \boldsymbol{\Phi})^{+} \mathbf{A} \boldsymbol{\Phi}$ in (22) can be predefined regardless of $\mathbf{d}_{l}$ or $\mathbf{c}_{l-1}$. Therefore, $N$-continuous symbol mapping is also data-independent, just like SVD precoding. It just requires that the information of each frequency-domain symbol is saved for its following symbol transmission. 
Since SVD precoding and $\mathrm{N}$-continuous symbol mapping are implemented in different places of the framework, both schemes can coexist in the system to keep both the null space property and the continuous derivative property. Let

$$
\mathbf{G}=\mathbf{G}^{S}, \hat{\mathbf{G}}=\left(\mathbf{G}^{S}\right)^{H}
$$

and

$$
\mathbf{c}_{l}=\mathbf{d}_{l}+\boldsymbol{\delta}_{l}^{N}, \hat{\mathbf{d}}_{l}=\hat{\mathbf{c}}_{l}-\hat{\boldsymbol{\delta}}_{l}^{N} .
$$

Note that (21) to (24) are based on the assumption that $\mathbf{G}$ is an identity matrix, so we just need to insert the SVD precoder $\mathbf{G}$ in (25) after $\boldsymbol{\Phi}$ from (21) to (24). Then, at the transmitter we can have

$$
\boldsymbol{\delta}_{l}^{N}=\left\{\begin{array}{c}
(\mathbf{A} \boldsymbol{\Phi} \mathbf{G})^{+} \mathbf{A c}_{l-1}-(\mathbf{A} \Phi \mathbf{G})^{+} \mathbf{A} \boldsymbol{\mathbf { T G }} \mathbf{G d}_{l}, l>1 \\
\mathbf{0}, l=1
\end{array}\right.
$$

Therefore,

$$
\mathbf{b}_{\mathbf{l}}=(\mathbf{A} \Phi \mathbf{G})^{+} \mathbf{A} \mathbf{b}_{l-1}+\left(\mathbf{G}-\mathbf{G}(\mathbf{A} \Phi \mathbf{G})^{+} \mathbf{A} \boldsymbol{\Phi} \mathbf{G}\right) \mathbf{d}_{l} .
$$

At the receiver, in the $i$ th $(i \geq 1)$ iteration, the estimated perturbation vector $\hat{\boldsymbol{\delta}}_{l}^{N,(i)}$

$$
\hat{\mathbf{\delta}}_{l}^{N,(i)}=(\mathbf{A} \boldsymbol{\Phi} \mathbf{G})^{+} \mathbf{A} \boldsymbol{\Phi} \mathbf{G} \overline{\mathbf{c}}_{l}-(\mathbf{A} \boldsymbol{\Phi} \mathbf{G})^{+} \mathbf{A} \boldsymbol{\Phi} \mathbf{G} \overline{\mathbf{d}}_{l}^{(i-1)},
$$

$\overline{\mathbf{d}}_{l}^{(i-1)}$ in (29) is supposed to be known. The $i$ th iteration decision vector $\hat{\mathbf{d}}_{l}^{(i)}$ is

$$
\begin{aligned}
\hat{\mathbf{d}}_{l}^{(i)}= & \left(\mathbf{I}-(\mathbf{A \Phi G})^{+} \mathbf{A} \Phi \mathbf{G}\right) \overline{\mathbf{c}}_{l} \\
& +(\mathbf{A} \boldsymbol{G})^{+} \mathbf{A} \boldsymbol{A} \mathbf{G} \overline{\mathbf{d}}_{l}^{(i-1)} .
\end{aligned}
$$

If it is not the last iteration, then $\overline{\mathbf{d}}_{l}^{(i)}(i \geq 1)$ is chosen from all possible frequency-domain symbol vectors (In other words, every entry of $\overline{\mathbf{d}}_{l}^{(i)}$ is a constellation point.) to minimize $\left\|\hat{\mathbf{d}}_{l}^{(i)}-\overline{\mathbf{d}}_{l}^{(i)}\right\|$. The initial vector $\overline{\mathbf{d}}_{l}^{(0)}=\mathbf{0}$. It is shown in [22] that the convergence is always achieved after just three iterations for an additive white Gaussian noise (AWGN) channel. However, the magnitude of the perturbation vector increases as $J$ increases, thus causing the degradation in BER. That is why a large $J$ should be avoided.

Again, if SVD precoding and $N$-continuous symbol mapping are combined without merging, the transmitted signal is either $\mathbf{F G}^{S}\left(\mathbf{d}_{l}+\boldsymbol{\delta}_{l}^{N}\right)$ or $\mathbf{F}\left(\mathbf{G}^{S} \mathbf{d}_{l}+\boldsymbol{\delta}_{l}^{N}\right)$. For the former case, the continuous property is not kept because $\mathbf{A} \boldsymbol{\Phi} \mathbf{G}^{S}\left(\mathbf{d}_{l}+\boldsymbol{\delta}_{l}^{N}\right) \neq \mathbf{A} \mathbf{b}_{l-1}$. For the latter case, the null space property is not kept because $\mathbf{P G}^{S} \mathbf{d}_{l}+\mathbf{P} \boldsymbol{\delta}_{l}^{N} \neq \mathbf{0}$. Therefore, combining but no merging, compared to implementing just one of them, would have the same effect with more cost, which is the same conclusion as in Section 4.1.

\subsection{Combination of SVD precoding [15] and filtering [8] (group $A$ and group $C$ )}

A filtering method is proposed in [8] to suppress the OOBE by inserting a transmitting filter $\mathbf{p}_{t x}=\left[p_{t x}[1] p_{t x}[2] \ldots p_{t x}\left[L_{p}\right]\right]^{T}$ in the time-domain approach block and a receiving filter $\mathbf{p}_{r x}=\left[p_{r x}[1] p_{r x}[2] \ldots p_{r x}\left[L_{p}\right]\right]^{T}$ in the time-domain recovery block, where $L_{p}$ is the filter length. Here the filter coefficients are derived by shifting a baseband filter $\mathbf{p}=\left[p[1] p[2] \ldots p\left[L_{p}\right]\right]^{T}:$

$$
p_{t x}[n]=p_{r x}[n]=p[n] e^{\frac{2 \pi f_{k} k^{n}}{F}}, n=1,2, \ldots, L_{p},
$$

where $n$ is the coefficient index, $f_{k}$ is the center subcarrier index of the passband, and $F$ is the IFFT size. $p$ $[n]$ is the $n$th sample of an equal ripple filter with passband attenuation of $0.75 \mathrm{~dB}$, stopband attenuation of $58 \mathrm{~dB}$, and stopband slope of 20 . The resulting transmit signal $\boldsymbol{x}_{l}$ is

$$
\mathbf{x}_{l}^{T}=\mathbf{p}_{t x}^{T} \otimes \mathbf{s}_{l}^{T},
$$

where $\otimes$ is the convolution operation. Using a matrix expression, we define

$$
\mathbf{H}=\left[\begin{array}{ccccccc}
p_{t x}[0] & 0 & \ldots & 0 & 0 & 0 & 0 \\
\vdots & p_{t x}[0] & 0 & \cdots & 0 & 0 & 0 \\
p_{t x}\left[L_{p}-2\right] & \vdots & p_{t x}[0] & 0 & \ddots & 0 & 0 \\
p_{t x}\left[L_{p}-1\right] & p_{t x}\left[L_{p}-2\right] & \vdots & p_{t x}[0] & 0 & \cdots & 0 \\
0 & p_{t x}\left[L_{p}-1\right] & p_{t x}\left[L_{p}-2\right] & \vdots & p_{t x}[0] & 0 & \vdots \\
\vdots & 0 & p_{t x}\left[L_{p}-1\right] & p_{t x}\left[L_{p}-2\right] & \vdots & \cdots & 0 \\
0 & \vdots & 0 & p_{t x}\left[L_{p}-1\right] & p_{t x}\left[L_{p}-2\right] & \vdots & p_{t x}[0] \\
0 & 0 & \vdots & 0 & p_{t x}\left[L_{p}-1\right] & \cdots & p_{t x}[1] \\
0 & 0 & 0 & \vdots & 0 & \ddots & \vdots \\
0 & 0 & 0 & 0 & \vdots & 0 & p_{t x}\left[L_{p}-1\right]
\end{array}\right]
$$

Then, we can rewrite (32) as

$$
\mathbf{x}_{l}=\mathbf{H} \boldsymbol{b}_{l} .
$$

The combination of the SVD precoding and the filtering is straightforward. The precoder $\mathbf{G}$ and the decoder $\widehat{\mathbf{G}}$ are given in (25). The filtering matrix $\mathbf{H}$ is given in (33). $\widehat{\mathbf{H}}$ can either be a pseudoinverse of $\mathbf{H}$ (i.e., an equalizer) or a match filter. No further change is needed.

Since the SVD precoding is a frequency-domain approach and the filtering is a time-domain approach, they have their fixed positions in our unified framework and are independent to each other if they are both implemented. Therefore, in this case, combining without merging is actually the same as the proposed combination, as we have also mentioned that no change is needed for the combined design. 


\subsection{Computational complexity analysis}

Now we analyze the computational complexity of the three hybrid schemes proposed above. They are named sequentially as hybrid $\mathrm{A}$, hybrid $\mathrm{B}$, and hybrid $\mathrm{C}$ schemes. For most of the schemes, the number of real multiplications at the transmitter is the same as that at the receiver except that the $\mathrm{N}$-continuous symbol mapping and hybrid B schemes depend on the number of iterations. Therefore, we define 'complexity' as the number of real multiplications required by an OFDM symbol to be transmitted. It is shown in [23] that, for an original OFDM system with $N$ subcarriers and $F$ IFFT inputs, the required complexity using the method called 'Split Radix FFT' is $F$ $\log _{2} F-3 F+4$. Then, for each precoding scheme, since the dimension of the precoding matrix is $N \times K$, the required number of real multiplications in the precoding process is $4 N K$, which has an order of $O\left(N^{2}\right)$. So, the complexity for either of the SVD, spectral, or hybrid A scheme is $F \log _{2} F-3 F+4+4 N K$. Next, for the $N$-continuous and hybrid $B$ schemes, the dimensions of $(\mathbf{A} \Phi)^{+} \mathbf{A}$ and $(\mathbf{A} \boldsymbol{\Phi})^{+} \mathbf{A} \boldsymbol{\Phi}$ in (22) are both $N \times N$, and the dimensions of $(\mathbf{A} \boldsymbol{\Phi} \mathbf{G})^{+} \mathbf{A}$ and $\mathbf{G}-\mathbf{G}(\mathbf{A} \boldsymbol{\Phi} \mathbf{G})^{+} \mathbf{A} \boldsymbol{\Phi} \mathbf{G}$ in (28) are $N \times N$ and $N \times K$, respectively. So, the complexity for the $N$ continuous scheme is $F \log _{2} F-3 F+4+8 N N$, which is much larger than the precoding schemes. The complexity for the hybrid B scheme is $F \log _{2} F-3 F+4+$ $4 N N+4 N K$, which is smaller than the $N$-continuous scheme. Finally, according to [8], $F \log _{2} F-3 F+4+F L_{p}+$ $\left(F+L_{\mathrm{CP}}\right) L_{p}$ is the complexity of the filtering scheme, where $L_{\mathrm{CP}}$ is the number of samples for CP. The complexity of the hybrid $\mathrm{C}$ scheme is the just precoding plus filtering, which is $F \log _{2} F-3 F+4+F L_{p}+\left(F+L_{\mathrm{CP}}\right) L_{p}+4 N K$. The complexity comparison is summarized in Table 4 with both the analytical expression and the numerical expression using the parameters defined later in Section 5.

\section{Numerical results}

In this section, first we present the PSD performance of the three combination examples in Section 4. We consider a 256-subcarrier OFDM system, i.e., let $N=256$. The subcarrier index $\left[\begin{array}{llll}f_{1} & f_{2} & \ldots & f_{256}\end{array}\right]$ are $\left[\begin{array}{llll}1 & 2 & \ldots & 256\end{array}\right]$. The QPSK modulation is used. The FFT size $F=1,024$. In the following figures, the PSD curves are obtained from applying the Fourier transform to a long sequence of time-domain symbols, taking the square, and then applying the smoothing process. The center frequency is shifted to zero, and the total bandwidth with 1,024 subcarriers is normalized to 1 .

Figures 2 and 3 show the PSD performance of the combination of SVD precoding and spectral precoding. $\mathrm{CP}$ is not inserted in Figure 2, i.e., $T_{\mathrm{CP}}=0$. In Figure 3, $T_{\mathrm{CP}} / T_{d}=9 / 128$. In these figures, the conventional OFDM result, which is denoted as the 'original' curve, is added as the reference. The 'SVD' curve means SVD precoding, The 'Spectral' curve means spectral precoding. The 'hybrid A' curve means the combination of these two precoding schemes. Note that the SVD and Spectral curves also can be the curves for the combination without merging. All three curves have the same overall coding rate, which is $K / N=248 / 256$, i.e., the redundancy $R=$ $N-K=8$. For the SVD and hybrid A curves, the number of the notched frequencies $M$ is 8 . Their indexes are chosen to be $[-34.5,-33.5,-32.5,-31.5,288.5,289.5$, 290.5, 291.5]. For the Spectral curve, $\mathbf{G}^{W_{8}}$ is used as the precoding matrix since it outperformed the other available precoding matrix $\mathbf{G}^{V_{8}}$ in our simulation. For the hybrid $\mathrm{A}$ curves, since the overall coding rate is $248 / 256$ (i.e., the overall redundancy $R$ is 8 ), we assign $248 / 255$ as the coding rate of the SVD precoding (i.e., its redundancy, defined as $R_{S}$, is 7) and $255 / 256$ as the coding rate of spectral precoding (i.e., its redundancy, defined as $R_{W}$, is 1 ) because this coding rate pair gave the best OOBE suppression among all the possible pairs in our simulation. As shown in Figure 4, the effectiveness (for OOBE suppression) per unit increment of redundancy of SVD precoding approximately remains the same (in terms of the unit $\mathrm{dB}$ ) as the number of redundancy $R_{S}$ increases linearly. On the other hand, in Figure 5, the effectiveness per unit increment of the redundancy for spectral precoding decreases drastically as the number of redundancy $R_{W}$ increases exponentially. In short, the OOBE suppression effect by spectral precoding is better than SVD precoding when the efficiency decreases from 1 to $(N-1) / N$. Then, the improvement from spectral precoding becomes less than SVD precoding when the efficiency decreases further from $(N-1) / N$. These two schemes have advantage at different

Table 4 The 'complexity' of the proposed schemes and their constituent schemes

\begin{tabular}{lcc}
\hline & $\begin{array}{c}\text { Complexity, the number of real multiplications } \\
\text { per symbol at the transmitter }\end{array}$ & $\begin{array}{c}\text { Complexity with the parameters } \\
\text { used in Figures 2, 3, 6, 7, 8, and 9 }\end{array}$ \\
\hline Original & $F \log _{2} F-3 F+4$ & 7,172 \\
SVD precoding, spectral precoding, hybrid $A$ & $F \log _{2} F-3 F+4+4 N K$ & 261,124 \\
$N$-continuous symbol mapping & $F \log _{2} F-3 F+4+8 N N$ & 531,460 \\
Hybrid B & $F \log _{2} F-3 F+4+4 N N+4 N K$ & 523,268 \\
Filtering & $F \log _{2} F-3 F+4+F L_{p}+\left(F+L_{C P}\right) L_{p}$ & 91,972 \\
Hybrid $C$ & $F \log _{2} F-3 F+4+F L_{p}+\left(F+L_{C P}\right) L_{p}+4 N K$ & 303,524 \\
\hline
\end{tabular}




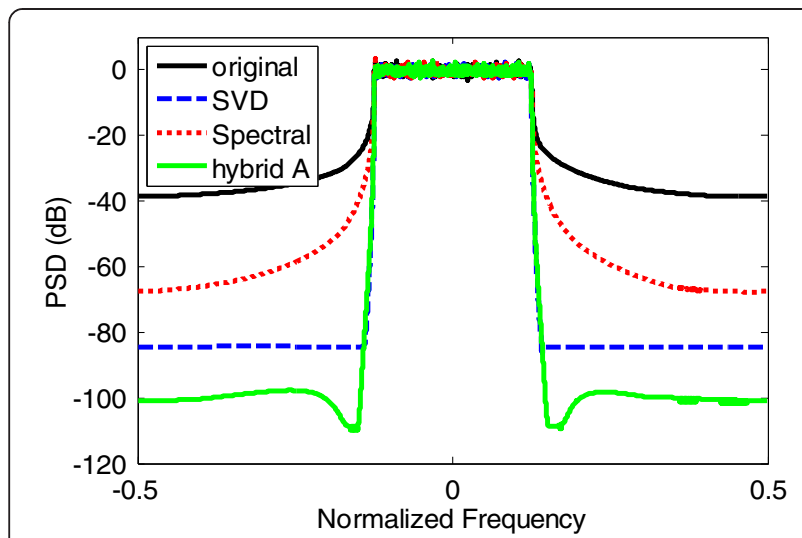

Figure 2 PSD of SVD precoding, spectral precoding, and their combination without $\mathrm{CP}$.

stages, so they are complementary to each other. By assigning one from the overall redundancy to the spectral precoding scheme (i.e., $R_{W}=1$ ) and the remaining to the SVD precoding scheme (i.e., $R_{S}=R-1$ ), we make sure that the complementary characteristics of both schemes are best exploited. Comparing the three precoded OFDM curves in Figure 2, we see that the combined scheme provides significant further improvement than both original schemes (or 'combined without merging' schemes) when they have the same overall coding rate.

Because the new basis functions used in spectral precoding have zero values only at finite number of locations, if $\mathrm{CP}$ is inserted, the new edges of an extended basis function are usually not zero and thus not be equal to the edges of its adjacent basis function. In other words, the continuous phase property cannot be maintained when CP is inserted. Therefore, in Figure 3, the Spectral curve shows very little improvement than the original curve. Moreover, the SVD curve in Figure 3 (with CP) also has worse OOBE suppression effect than the SVD curve in Figure 2 (without CP). This is due to the fact that when $\mathrm{CP}$ is not inserted, the

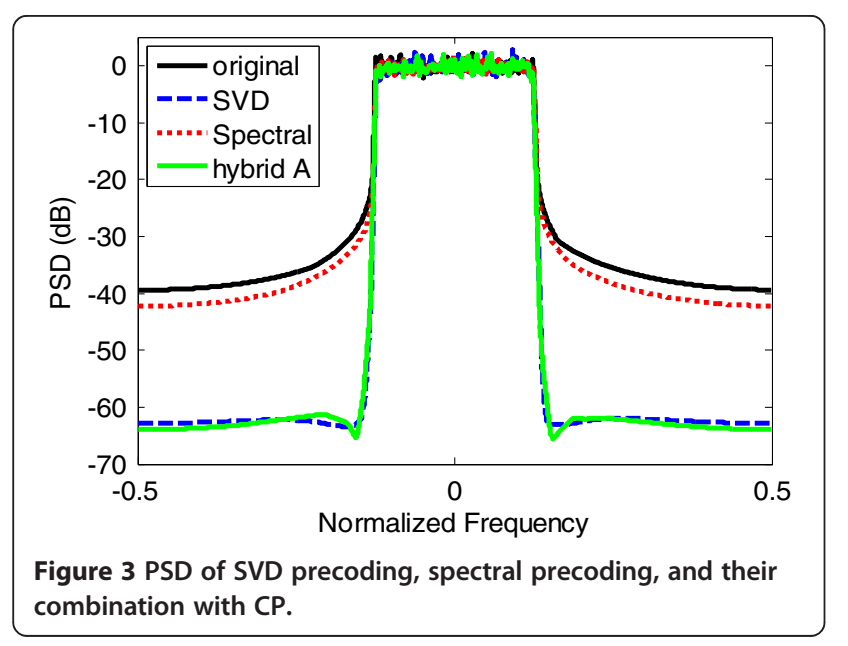

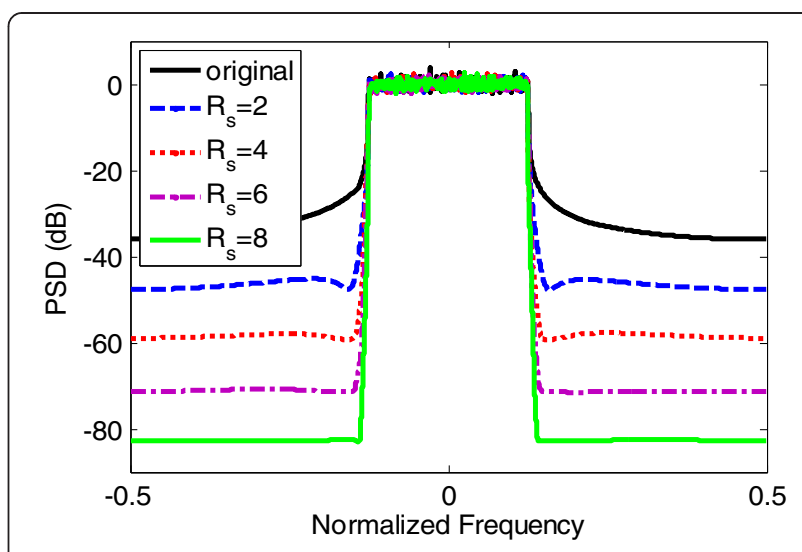

Figure 4 PSD of SVD precoding with different $R_{S}$ without CP.

peak of a sidelobe from a subcarrier aligns in frequency with the peak of a certain sidelobe from each of all other subcarriers. Thus, each notch frequency we use here $(0.5$ plus an integer) suppresses the peak of a certain sidelobe from each of all subcarriers. But when $\mathrm{CP}$ is inserted, the sidelobes from different subcarriers do not align well anymore, and the effects of notch frequencies for suppressing sidelobes are reduced. Nevertheless, the SVD precoding scheme in the with-CP case is still quite effective in suppressing OOBE. Since the spectral precoding scheme is not effective when there is $\mathrm{CP}$, the hybrid A and the SVD precoding have similar performances.

In summary, compared with the original SVD precoding and spectral precoding schemes, the combined scheme has no extra drawback, such as the same computational complexity, the same PAPR, and the same BER (which will be shown later in the paper), because the dimension of the precoding matrix is never changed by the process of combining. It provides significant further improvement on OOBE suppression than either of these two original schemes without $\mathrm{CP}$ insertion. However, when there is $\mathrm{CP}$




insertion, the spectral precoding is not useful. Fortunately, the SVD precoding is still quite effective (although it is not as effective as in the case of without CP). Therefore, hybrid A is not necessary and just SVD precoding is good enough with CP insertion. To this regard, hybrid A would be useful in zero padding (ZP) OFDM systems where CP is not required [24].

Figures 6 and 7 show the PSD performance of the combination of SVD precoding and $N$-continuous symbol mapping. In Figure 6, $T_{\mathrm{CP}}=0$. In Figure 7, $T_{\mathrm{CP}} / T_{d}=9 / 128$. The ' $\mathrm{N}$-con 1 ' curve means $N$-continuous symbol mapping with $J=1$. The 'N-con 4' curve means $N$-continuous symbol mapping with $J=4$. The 'hybrid B 1' curve means the combination of SVD precoding and $N$-continuous symbol mapping with $J=1$. The 'hybrid B 2' curve means the combination of SVD precoding and $N$-continuous symbol mapping with $J=2$. The coding rates and the notch frequencies for the 'SVD' and 'hybrid B' curves are the same as those for the curves in Figures 2 and 3 . Note that the SVD, N-con 1 , and N-con 4 curves also can be the curves for the combination without merging.

In Figure 6, the N-con 1 and $\mathrm{N}$-con 4 curves have slow roll-off and large transition bands, while the SVD curve has a much smaller transition band. Compared to these two schemes, the combined scheme has a small transition band as in the SVD scheme while providing very good OOBE suppression. This is because these two existing schemes suppress the OOBE by using two properties independent of each other, which are the null space property and the continuous derivative property. These two properties are both kept in the combined scheme. An advantage of the hybrid B scheme is that it is able to achieve better OOBE suppression with a much smaller value of $J$ compared with the constituent $N$-continuous scheme. By comparing the $\mathrm{N}$-con 4 curve with the hybrid B 1 curve, we can see that the hybrid B scheme with $J=1$ has a smaller transition band and

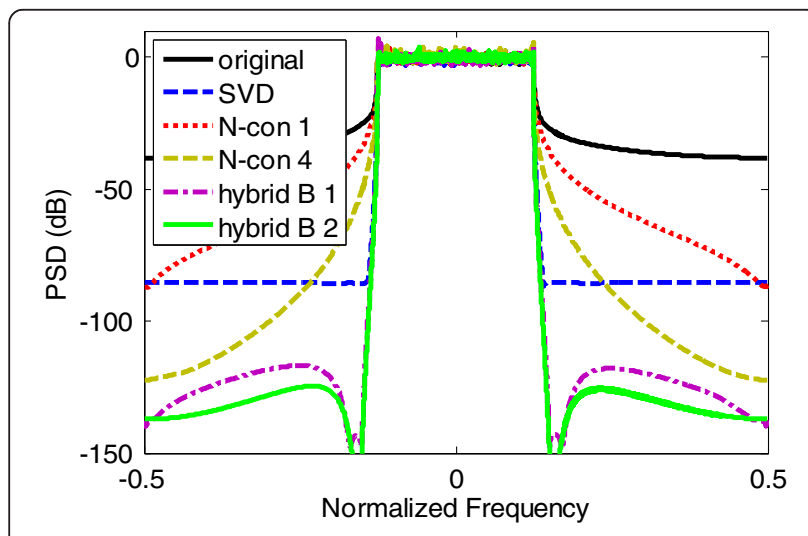

Figure 6 PSD of SVD precoding, $\mathrm{N}$-continuous symbol mapping, and their combination without $\mathrm{CP}$.

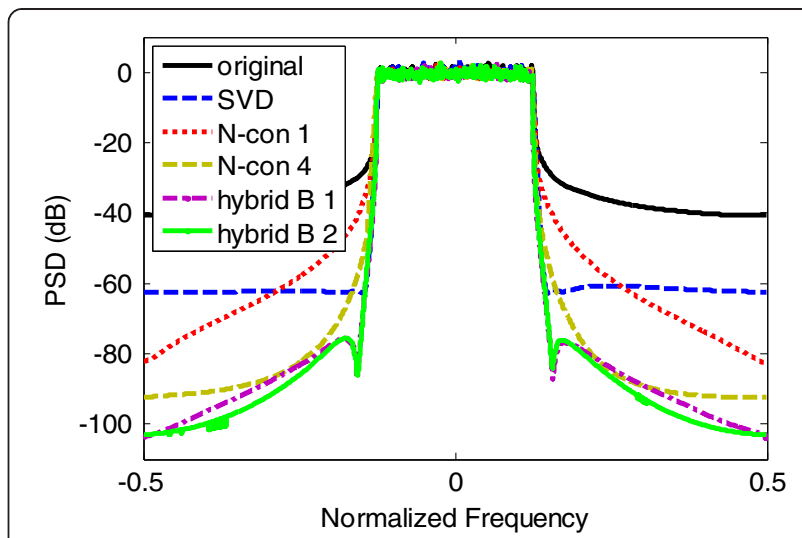

Figure 7 PSD of SVD precoding, $\mathrm{N}$-continuous symbol mapping, and their combination with $\mathrm{CP}$.

larger OOBE suppression than the original $N$-continuous scheme even with $J=4$. The hybrid B 2 are put as the reference. The improvement from increasing $J$ for the hybrid $\mathrm{B}$ scheme is insignificant because the OOBE is already very low. Note that the dips as low as $-150 \mathrm{~dB}$ for SVD in Figure 6 are at the null frequencies. They are not artifacts due to constraint on numerical precision because the PSD curves have already been smoothened. In Figure 7, although the SVD curve is affected by CP insertion as discussed previously, it is still quite effective in OOBE suppression, while the $\mathrm{N}$-con 1 and $\mathrm{N}$-con 4 curves are unaffected in their transition bands no matter with or without CP. The hybrid B 1 curve in Figure 7 still has the small transition while providing better OOBE suppression just like the case without $C P$ in Figure 6. It is shown in [22] that this combined scheme gives no extra degradation in the system performances such as the increase of BER or PAPR compared to either $N$-continuous symbol mapping or SVD precoding by the usage of a small maximum derivative order and the iterative decoder at the receiver. In conclusion, the proposed hybrid B scheme is well suitable for the CP-OFDM scenario.

Figures 8 and 9 show the PSD performance of the combination of SVD precoding and filtering. In Figure 8, $T_{\mathrm{CP}}=0$. In Figure 9, $T_{\mathrm{CP}} / T_{d}=9 / 128$. The 'Filter' curve means the filtering scheme. The 'hybrid C' curve means the combination of SVD precoding and filtering. For the 'SVD' and hybrid C curves, the number of the notched frequencies $M$ is 12 . Their indexes are chosen to be $[-51.5,-50.5,-32.5,-31.5,-11.5,-10.5,267.5,268.5$, $288.5,289.5,307.5,308.5]$. The redundancy is $R=8$. For the Filter curve, the filter length $L p$ is 40 . For the hybrid $\mathrm{C}$ curve, the filter length is 20 . The spectrum efficiencies for the four curves are the following: Let the number of samples for CP, $L_{\mathrm{CP}}=F \times T_{C P} / T_{d}$. Then, for the 'original' curve, the spectrum efficiency is $1-L_{\mathrm{CP}} /\left(L_{\mathrm{CP}}+F\right)=$ 0.9343. For the SVD curve, the spectrum efficiency is 


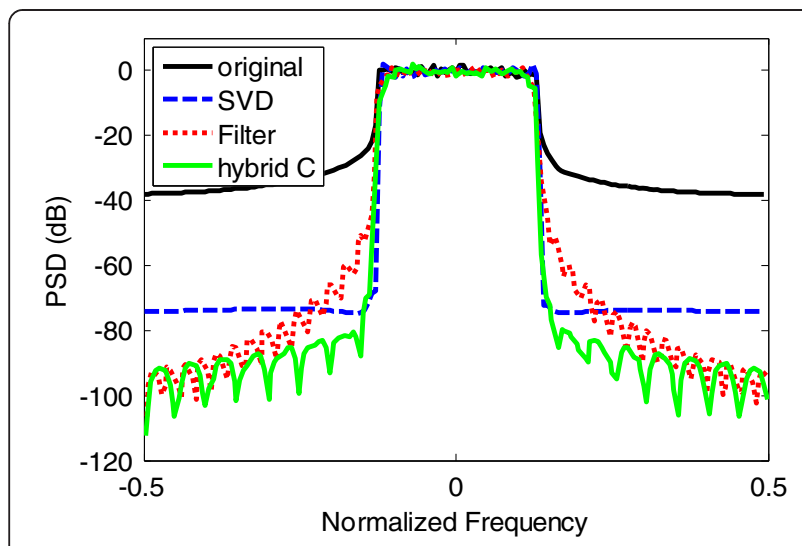

Figure 8 PSD of SVD precoding, filtering, and their combination without $\mathrm{CP}$.

$248 / 256 \times\left(1-L_{\mathrm{CP}} /\left(L_{\mathrm{CP}}+F\right)\right)=0.9051$. For the Filter curve, the spectrum efficiency is $\left(1-L_{\mathrm{CP}} /\left(L_{\mathrm{CP}}+F+L_{P}-1\right)\right)=$ 0.9022 . For the hybrid $C$ curve, the spectrum efficiency is $\left(1-L_{\mathrm{CP}} /\left(L_{\mathrm{CP}}+F+L_{P}-1\right)\right) \times 248 / 256=0.9040$. Here, for the calculation of spectral efficiencies, we assume that the $\mathrm{CP}$ length is increased because of the filters used in both hybrid $\mathrm{C}$ and filtering schemes. Thus, we choose the parameters such that the spectral efficiencies of the three OOBE suppression schemes are more or less the same. Note that the hybrid $\mathrm{C}$ curve is also the curve for the combination without merging.

As mentioned before, SVD precoding is still quite effective in OOBE suppression when there is $\mathrm{CP}$ although the result degrades. On the contrary, the filtering scheme does not depend much on whether there is $\mathrm{CP}$ or not. Thus, the Filter curves in both Figures 8 and 9 look similar, and they can achieve larger stopband attenuation but slower roll-off than the SVD curves. In both Figures 8 and 9, the hybrid $\mathrm{C}$ curves can achieve higher stopband attenuation than SVD curves and quicker roll-off and smaller

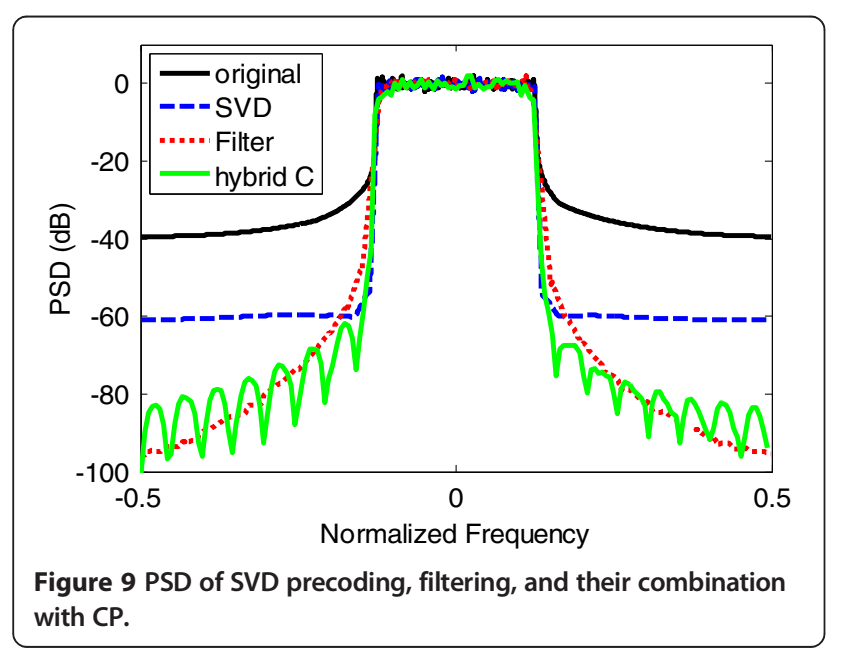

transition band than Filter curses. Thus, the hybrid C scheme has the advantages of both the SVD precoding scheme (i.e., quick roll-off or transition) and the filtering scheme (i.e., large stopband attenuation).

Next, we take a brief look at the PAPR and the BER issues. As been observed previously in [18], SVD precoding and spectral precoding do not change the BER but increase the PAPR slightly. The lower the coding rate is, the higher the PAPR is. Van de Beek and Berggren [17] show that $N$-continuous symbol mapping does not change either the PAPR or the BER, provided that the maximum derivative order is very small (which is one here) and a sufficient number of iterations (which is three here) is used in the receiver. The filtering does not change the PAPR but increases the BER slightly, which is shown in [25].

Figure 10 shows the complementary cumulative distribution function (CCDF) of the eight schemes without CP insertion. The overall coding rate is $248 / 256$ for all precoding and hybrid schemes. In Figure 10, the 'original', 'N-con', and 'Filter' curves overlap together, and they are slightly lower than the other five overlapping curves. For the hybrid A scheme, due to the same overall coding rate, it has the same PAPR as the SVD and spectral precoding schemes. So are the hybrid $B$ and $C$ schemes because $\mathrm{N}$-continuous symbol mapping and filtering do not change PAPR. However, for the hybrid C scheme, if the same spectrum efficiency loss rather than the same coding rate is assumed, as filtering takes a part of the total spectrum efficiency loss, the hybrid C scheme would have lower PAPR than the other precoding schemes, which is shown later in Table 5.

Figure 11 shows the BER performance. An AWGN channel is assumed. $E_{b} / N_{o}$ in the abscissa stands for the energy per information bit divided by the average noise power density. QPSK modulation is used. The overall coding rate is $248 / 256$ for all precoding and hybrid

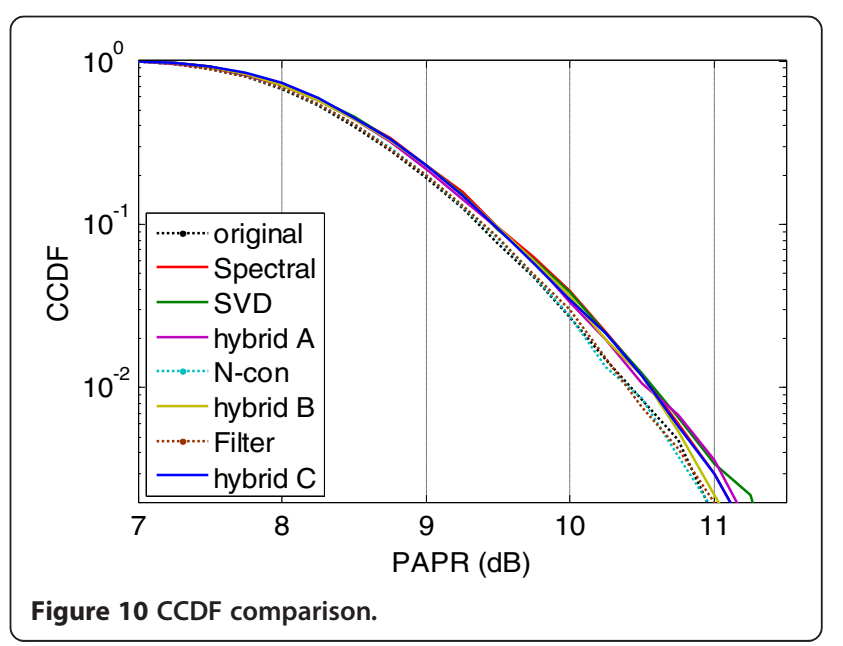


Table 5 The proposed schemes vs. the SVD precoding scheme under the same spectrum efficiency loss

\begin{tabular}{llll}
\hline & OOBE suppression & PAPR & BER \\
\hline Hybrid A & $\begin{array}{l}\text { Much better without } \\
\text { CP; same with CP }\end{array}$ & Same & Same \\
Hybrid B & Much better & Same & $\begin{array}{l}\text { Same with enough iterations } \\
\text { (3 in our simulation) }\end{array}$ \\
Hybrid C & Better & Better & Worse \\
\hline
\end{tabular}

schemes. The filter length $L p$ is 40 . We can see that all the schemes except 'Filter' and 'hybrid C' have exactly the same BER performance. This is because none of their constituent schemes changes the BER. The Filter and hybrid $\mathrm{C}$ curves overlap with the other curves in the beginning and then slightly diverge when $E_{b} / N_{o}$ becomes large. Since the filter suppresses the data from the subcarriers near the edges at the transmitter, then the equalizer at the receiver needs to compensate by doing the amplification. Therefore, the noise from these subcarriers is also amplified.

The PAPR and BER comparison is also shown in Table 5, where three hybrid schemes are compared with their common constituent scheme, SVD precoding, when all of them have the same spectrum efficiency loss. In the figures, we show that SVD precoding is able to reach $-100 \mathrm{~dB}$ OOBE suppression and any other value smaller than $100 \mathrm{~dB}$ on its own. The cost, however, is the loss of the spectrum efficiency. We have shown that the combination of SVD precoding and spectral precoding can reach the same goal at the cost of a smaller loss of the spectral efficiency in scenarios without cyclic prefix. We have also shown that the other two hybrid schemes reduce the cost regardless of whether cyclic prefix is inserted or not. Moreover, since the lower coding rate causes higher PAPR, which is observed previously, the hybrid schemes also have lower PAPR than SVD precoding only to achieve the same OOBE suppression goal, thus reducing the distortion brought by the

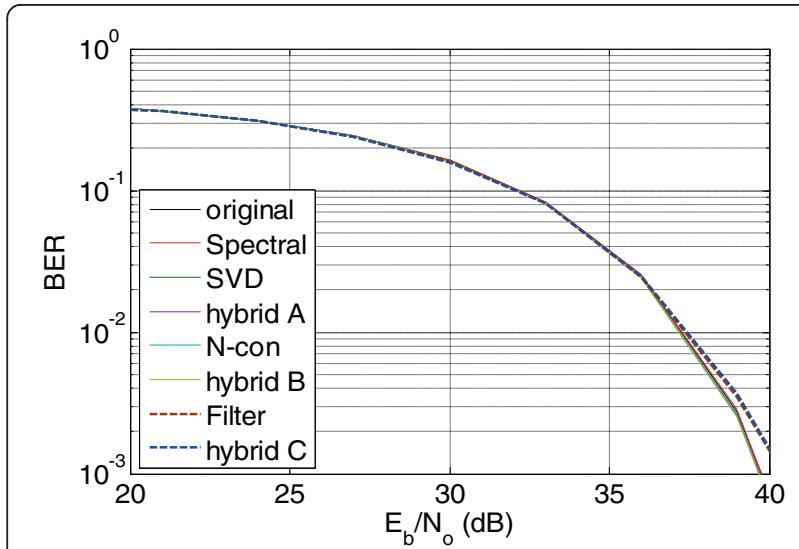

Figure 11 BER comparison. non-linearity of the power amplifier. From Table 5, we conclude that hybrid $\mathrm{B}$ is preferred in the $\mathrm{CP}$ scenario (with an increased complexity) and hybrid $\mathrm{A}$ is preferred in the without-CP scenario (without any complexity increase) for practical applications.

\section{Conclusion}

In this paper, we first present a generalized OFDM framework which can always describe an OFDM system no matter whether any of OOBE suppression schemes is applied. Then, we briefly investigate some existing OOBE suppression techniques and classify them into three groups, which are symbol mapping techniques, precoding techniques, and time-domain techniques, depending on the positions where they are embedded in our framework. Once all the OOBE suppression designs are based on the same unified system, it becomes very convenient for us to judge whether different schemes are suitable or not if they are both implemented simultaneously in one system and then perform the combining designs for those compatible schemes. To show the benefits of using this framework, we propose three types of data-independent hybrid OOBE suppression schemes as the examples, which are the combinations of SVD precoding and other three techniques belonging to three different groups, namely spectral precoding (group A), $\mathrm{N}$-continuous symbol mapping (group $\mathrm{B})$, and filtering (group C), respectively.

Numerical results show that all three proposed hybrid schemes have better OOBE suppression effects than the original schemes and the improvements are more or less depending on the level of their compatibility and the performance of the original schemes. The combination of SVD precoding and spectral precoding is ideal for the systems where CP is not inserted (such as ZP-OFDM systems) because the continuous phase property brought by the spectral precoding scheme still can be maintained after zero padding. Since both original schemes belong to the same group, their combination does not give any extra drawback than either of original schemes when the combined scheme and the two original schemes have the same coding rate. The other two combined schemes are well suitable for CP-OFDM systems. This is because neither the $N$-continuous symbol mapping scheme nor the filtering scheme depends on whether there is $\mathrm{CP}$ or not, and SVD precoding is still quite effective even though it is affected by the CP insertion. In both scenarios with and without $\mathrm{CP}$, these two combined schemes provide both the small transition band (from SVD precoding) and the large suppression in the stopband (from $\mathrm{N}$-continuous symbol mapping or filtering).

In addition to comparing the hybrid schemes with the individual constituent schemes, we also include the case that original schemes are implemented in a decentralized way that each scheme is designed without knowing 
the existence of the other scheme. For hybrid A and B schemes, their corresponding 'combining without merging' schemes are even worse than the original schemes considering the extra cost of spectrum efficiency. For the hybrid $\mathrm{C}$ scheme, the combination without merging is the same as with merging. Just because of the fact that applying different methods in one system causes different results, a unified framework is required, which our classification of existing OOBE suppression techniques is based on. With the help of such a framework, a system can still be analytically described after multiple schemes are applied simultaneously. Therefore, it is easy to check whether the required condition for a scheme to work is still satisfied when this scheme is applied together with other schemes, and it is easy to do the joint design so that the requirements for different schemes can be kept as all of the applied schemes are viewed as a single new scheme.

Besides the sidelobes which are generated due to the structure of the OFDM transmitter, the signal distortion that is caused by a non-linear transmitter, e.g., the intermodulation distortion (IMD), is indeed another cause of OOBE besides the sidelobes. The IMD reduction techniques are not investigated in our paper since the system is assumed to be linear. However, if the information of the non-linear system is known (for example, the parameters of a non-linear power amplifier), then the current framework could be extended by inserting a 'non-linear system' module after the 'time-domain approaches' module in Figure 1. Some of the IMD reduction techniques are similar to the OOBE reduction techniques. For example, coding, selective mapping, and partial transmit sequence methods can be used for both purposes above, which is shown in $[26,27]$. Therefore, for these techniques, such an extended framework might be used to jointly reduce the OOBE from sidelobes and IMD. On the other hand, we know that the transmitted signals of large PAPR require the power amplifier to have a large linear range, thus being more likely to generate larger OOBE than the signals of small PAPR. Some of the PAPR reduction methods can be classified in the same way into one of the three groups we proposed for the classification of OOBE suppression techniques. For example, some precoding techniques in $[28,29]$ can be viewed as the group $\mathrm{A}$ techniques that are represented by precoding matrices. The tone injection method in [30] can be viewed as a group B technique that is represented by symbol mapping. These PAPR reduction techniques above can be combined with the OOBE suppression techniques in our proposed framework to jointly reduce the PAPR and OOBE. However, some other PAPR reduction techniques, such as clipping and companding [31], do not belong to any of the groups and cannot be described by our framework so far. Moreover, some methods reduce the PAPR by distorting the signal, thus increasing the OOBE. It may not be a good idea to implement one of these non-linear methods and an OOBE suppression scheme in one system. In short, to develop an OFDM framework for some more general purpose besides suppressing the OOBE from sidelobes and to develop hybrid techniques for jointly suppressing OOBE from both sidelobes and non-linear distortion are our future work.

\section{Competing interests}

The authors declare that they have no competing interests.

\section{Acknowledgements}

The work is supported in part by InterDigital Communications, Inc.

Received: 13 September 2013 Accepted: 29 April 2014

Published: 19 May 2014

\section{References}

1. J Mitola, GQ Maguire Jr, Cognitive radio: making software radios more personal. IEEE Pers. Commun. 6(4), 13-18 (1999)

2. T Weiss, FK Jondral, Spectrum pooling: an innovative strategy for the enhancement of spectrum efficiency. IEEE Commun. Mag. 42(3), 8-14 (2004)

3. F Meucci, O Cabral, FJ Velez, A Mihovska, NR Prasad, Spectrum aggregation with multi-band user allocation over two frequency bands, in Proc. IEEE Mobile WiMAX Symp (Nappa Valley, 2009). 81-86 Jul

4. C Cordeiro, K Challapali, D Birru, IEEE 802.22: an introduction to the first wireless standard based on cognitive radios. J. Commun. 1, 1 (2006)

5. Y Wu, WY Zou, Orthogonal frequency division multiplexing: a multi-carrier modulation scheme. IEEE Trans. Consum. Electron. 41(3), 392-399 (1995)

6. SM Alamouti, A simple transmit diversity technique for wireless communications. IEEE J. Sel. Areas Commun. 16(8), 1451-1458 (1998)

7. U Berthold, FK Jondral, Guidelines for designing OFDM overlay systems, in Proc. 1st IEEE Symp. New Frontiers Dyn (Spectrum Access Netw, Baltimore, 2005), pp. 626-629

8. D Noguet, M Gautier, V Berg, Advances in opportunistic radio technologies for TWWS. EURASIP J. Wirel. Commun. Netw 2011, 170 (2011)

9. T Weiss, J Hillenbrand, A Krohn, FK Jondral, Mutual interference in OFDM based spectrum pooling systems. Proc. IEEE Vehicular Technol. Conference (VTC) 4, 1873-1877 (2004)

10. MS El-Saadany, AF Shalash, M Abdallah, Revisiting active cancellation carriers for shaping the spectrum of OFDM-based cognitive radios, in Proc. IEEE Sarnoff Symposium (SARNOFF), 2009, pp. 1-5

11. A Sahin, H Arslan, Edge windowing for OFDM based systems. IEEE Commun. Lett. 15(11), 1208-1211 (2011)

12. S Brandes, I Cosovic, M Schnell, Reduction of out-of-band radiation in OFDM systems by insertion of cancellation carriers. IEEE Commun. Lett. 10(6), 420-422 (2006)

13. S Pagadarai, R Rajbanshi, AM Wyglinski, GJ Minden, Sidelobe suppression for OFDM-based cognitive radios using constellation expansion, in Proc. IEEE Wireless Communications and Networking Conference (WCNC) (Las Vegas, 2008), pp. 888-893

14. J van de Beek, Orthogonal multiplexing in a subspace of frequency well-localized signals. IEEE Commun. Lett. 14(10), 882-884 (2010)

15. M Ma, X Huang, B Jiao, YJ Guo, Optimal orthogonal precoding for power leakage suppression in DFT-based systems. IEEE Trans. Commun. 59(3), 844-853 (2011)

16. C-D Chung, Spectrally precoded OFDM. IEEE Trans. Commun. 54(12), 2173-2185 (2006)

17. J van de Beek, F Berggren, N-continuous OFDM. IEEE Commun. Lett. 13, 1-3 (2009)

18. Z You, J Fang, I-T Lu, Combination of spectral and SVD precodings for out-of-band leakage suppression, in IEEE Long Island Systems, Applications and Technology Conference (LISAT) (Farmingdale, 2013)

19. I Cosovic, S Brandes, M Schnell, Subcarrier weighting: a method for sidelobe suppression in OFDM systems. IEEE Commun. Lett. 1(6), 444-446 (2006)

20. I Cosovic, T Mazzoni, Suppression of sidelobes in OFDM systems by multiple-choice sequences. Eur Trans. Telecommun. 17(6), 623-630 (2006) 
21. HA Mahmoud, H Arslan, Sidelobe suppression in OFDM-based spectrum sharing systems using adaptive symbol transition. IEEE Commun. Lett. 12(2), 133-135 (2008)

22. Z You, J Fang, I-T Lu, Hybrid combination of $\mathrm{N}$-continuous and null-space precoding for out-of-band emission suppression, in Military Communications Conference (Milcom) (San Diego, 2013), pp. 1576-1580

23. HS Malvar, Signal Processing With Lapped Transforms (Artech House, London, 1992)

24. B Muquet, Z Wang, GB Giannakis, M de Courville, P Duhamel, Cyclic prefixing or zero padding for wireless multicarrier transmissions? IEEE Trans. Commun. 50(12), 2136-2148 (2002)

25. J Li, E Bala, R Yang, Resource block filtered-OFDM for future spectrally agile and power efficient systems. Phys. Commun. (2013). in press

26. MRD Rodrigues, IJ Wassell, IMD reduction coding to improve the error probability performance of nonlinearly distorted OFDM signals. IEE Proc. Commun. 152(5), 559-566 (2005)

27. MRD Rodrigues, IJ Wassell, IMD reduction with SLM and PTS to improve the error-probability performance of nonlinearly distorted OFDM signals. Proc. IEEE Vehicular Technol. Conference (VTC) 55(2), 537-548 (2006)

28. SB Slimane, Reducing the peak-to-average power ratio of OFDM signals through precoding. IEEE Trans. Veh. Technol. 56(2), 686-695 (2007)

29. M Zhang, Z Wang, Joint improved Nyquist pulse shaping and clipping for PAPR reduction of OFDM signals, in Proc. Wireless Communications, Networking and Mobile Computing (WiCOM) (Wuhan, 2011)

30. J Tellado, JM Cioffi, Peak power reduction for multicarrier transmission, in Proc. IEEE GlobeCom, 1998, pp. 219-224

31. SH Han, JH Lee, An overview of peak-to-average power ratio reduction techniques for multicarrier transmission. IEEE Trans. Wireless Commun. 12(2), 56-65 (2005)

doi:10.1186/1687-6180-2014-74

Cite this article as: You et al:: Out-of-band emission suppression

techniques based on a generalized OFDM framework. EURASIP Journal on Advances in Signal Processing 2014 2014:74.

\section{Submit your manuscript to a SpringerOpen ${ }^{\circ}$ journal and benefit from:}

- Convenient online submission

- Rigorous peer review

- Immediate publication on acceptance

- Open access: articles freely available online

- High visibility within the field

- Retaining the copyright to your article

Submit your next manuscript at $\gg$ springeropen.com 